\title{
Link between superconductivity and a Lifshitz transition in intercalated $\mathrm{Bi}_{2} \mathrm{Se}_{3}$
}

\author{
A. Almoalem, ${ }^{1}$ I. Silber $\odot,{ }^{2}$ S. Sandik, ${ }^{2}$ M. Lotem $\odot,{ }^{2}$ A. Ribak $\odot,{ }^{1}$ Y. Nitzav,${ }^{1}$ A. Yu. Kuntsevich $\odot,{ }^{3}$ O. A. Sobolevskiy $\odot,{ }^{3}$ \\ Yu. G. Selivanov ${ }^{1},{ }^{3}$ V. A. Prudkoglyad, ${ }^{3}$ M. Shi, ${ }^{4}$ L. Petaccia $\odot,{ }^{5}$ M. Goldstein $\odot,{ }^{2}$ Y. Dagan $\odot,{ }^{2}$ and A. Kanigel ${ }^{1}$ \\ ${ }^{1}$ Department of Physics, Technion - Israel Institute of Technology, Haifa 32000, Israel \\ ${ }^{2}$ School of Physics and Astronomy, Tel Aviv University, Tel Aviv 6997801, Israel \\ ${ }^{3}$ P. N. Lebedev Physical institute, RAS, Moscow 119991, Russia \\ ${ }^{4}$ Swiss Light Source, Paul Scherrer Institute, CH-5232 Villigen, Switzerland \\ ${ }^{5}$ Elettra Sincrotrone Trieste, Strada Statale 14 km 163.5, I-34149 Trieste, Italy
}

(Received 21 December 2020; revised 30 April 2021; accepted 7 May 2021; published 26 May 2021)

\begin{abstract}
Topological superconductivity is an exotic phase of matter in which the fully gapped superconducting bulk hosts gapless Majorana surface states protected by topology. Intercalation of copper, strontium, or niobium between the quintuple layers of the topological insulator $\mathrm{Bi}_{2} \mathrm{Se}_{3}$ increases the carrier density and leads to superconductivity that is suggested to be topological. Here we study the electronic structure of strontium-intercalated $\mathrm{Bi}_{2} \mathrm{Se}_{3}$ using angle-resolved photoemission spectroscopy and Shubnikov-de Haas oscillations. Despite the apparent low Hall number of $\sim 2 \times 10^{19} \mathrm{~cm}^{-3}$, we show that the Fermi surface has the shape of an open cylinder with a larger carrier density of $\sim 10^{20} \mathrm{~cm}^{-3}$. We suggest that superconductivity in intercalated $\mathrm{Bi}_{2} \mathrm{Se}_{3}$ emerges with the appearance of a quasi-two-dimensional open Fermi surface.
\end{abstract}

DOI: 10.1103/PhysRevB.103.174518

\section{INTRODUCTION}

A promising avenue towards achieving topological superconductivity is to induce superconductivity in materials whose electronic structure is topologically nontrivial [1]. Intercalated $\mathrm{Bi}_{2} \mathrm{Se}_{3}$ has been suggested as a possible candidate for a topological superconductor based on the appearance of a zero-bias feature in the tunneling conductance [2-5] and nematicity in the superconducting state [5-12]. A possible explanation of the zero-bias peak is the existence of Majorana modes $[2,4,5]$. Also, nematicity can be accounted for by a two-component Ginzburg-Landau theory with nematic solutions corresponding to topological superconductivity with odd-parity $E_{u}$ symmetry (the order parameter is odd under the operations $x \rightarrow-x$ and $y \rightarrow-y$ ). Such nematic solutions require an open, quasi-two-dimensional (quasi-2D), Fermi surface [13-16].

A simple criterion has been put forth for determining whether superconductivity in $\mathrm{Bi}_{2} \mathrm{Se}_{3}$-derived systems is three-dimensional (3D) topological time reversal invariant (TRI): the Fermi surface (FS) has to enclose an odd number of time-reversal-invariant momenta (TRIM) points [17]. Superconducting $A_{x} \mathrm{Bi}_{2} \mathrm{Se}_{3}$ compounds are slightly electron doped $\left(n \sim 10^{19}-10^{20} \mathrm{~cm}^{-3}\right)$, with the bottom of the conduction band located at the center of the Brillouin zone [18]. In $\mathrm{Cu}_{x} \mathrm{Bi}_{2} \mathrm{Se}_{3}$, angle-resolved photoemission spectroscopy (ARPES), Shubnikov-de Hass (SdH), and de Haas-van Alphen $[19,20]$ experiments found an open FS that encloses two TRIM points ( $\Gamma$ and $Z$ ), thus not fulfilling the aforementioned criterion but suggesting the possibility of weak 2D topological superconductivity.

Increasing the carrier density of $\mathrm{Bi}_{2} \mathrm{Se}_{3}$ via $\mathrm{Cu}$ intercalation results in a quick expansion of the FS towards the $Z$ point and, eventually, a Lifshitz transition into an open cylindrical-like
FS [19]. Interestingly, Sr-intercalated $\mathrm{Bi}_{2} \mathrm{Se}_{3}$ is reported to have an order of magnitude smaller carrier concentration than the $\mathrm{Cu}$ intercalation while still exhibiting superconductivity with similar critical temperature $T_{c}[7,21,22]$. This raises two questions: (1) Does $\mathrm{Sr}_{x} \mathrm{Bi}_{2} \mathrm{Se}_{3}$ have a closed Fermi surface that allows for a 3D topological TRI superconductivity? (2) How can such a small number of carriers produce $T_{c} \simeq 3 \mathrm{~K}$ ?

In this paper we combine the SdH effect and ARPES measurements to determine the shape of the Fermi surface, motivated by the relatively small Hall number of $\mathrm{Sr}_{x} \mathrm{Bi}_{2} \mathrm{Se}_{3}$. We study $\mathrm{Sr}_{x} \mathrm{Bi}_{2} \mathrm{Se}_{3}$ samples with $x_{\text {nominal }}=0.15$; crystal growth and characterization are described in Refs. [7,23] and in Appendix A. The samples display Dirac-like surface states as well as a parabolic bulk band. A naive estimate of the carrier density from the Hall slope yields a carrier concentration of $\sim 2 \times 10^{19} \mathrm{~cm}^{-3}$. This estimate is in good agreement with the binding energy of the Dirac point [19] but has a noticeable temperature dependence (see Fig. 1). Bulk superconductivity is evident from the large $\mathrm{SC}$ volume fraction, which extrapolates to $100 \%$ at zero temperature, with $T_{c}=2.7 \mathrm{~K}$.

\section{RESULTS AND DISCUSSION}

\section{A. Shubnikov de Hass measurements}

The frequency of the resistance oscillations as a function of inverse magnetic field corresponds to the cross-sectional areas of the extrema of the FS perpendicular to the magnetic field [24]. We therefore rotate the magnetic field with respect to the $c$ axis $\theta$ to resolve the shape of the FS. Our main finding using this method is the clear observation of two frequencies when the magnetic field is parallel to the $c$ axis, $\theta=0$ [see Fig. 2(a)]. 

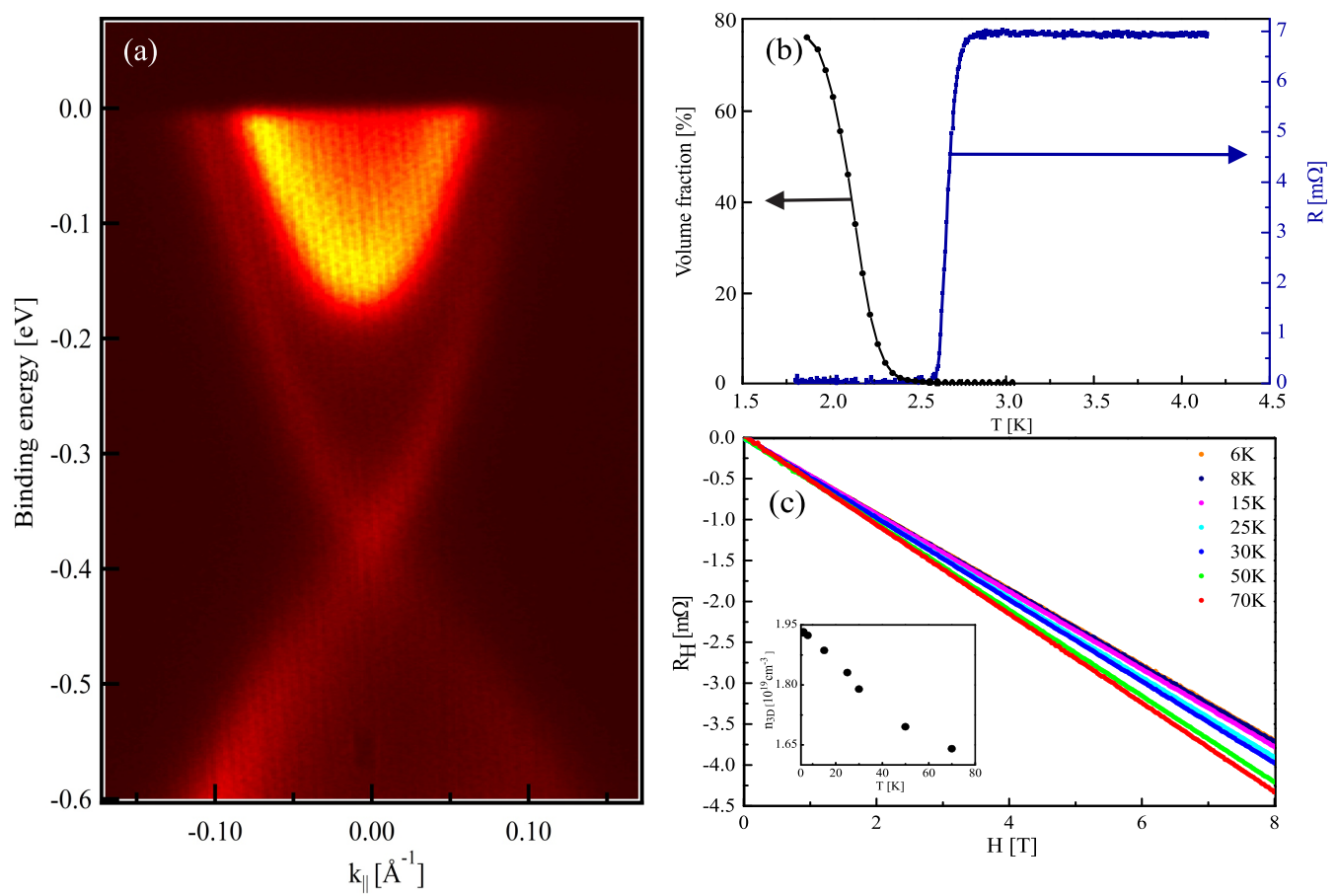

FIG. 1. Bulk superconductivity coexists with topological surface states in $\mathrm{Sr}_{x} \mathrm{Bi}_{2} \mathrm{Se}_{3}$. (a) Typical ARPES spectrum, taken using a 20 eV photon energy at $T=28.5 \mathrm{~K}$, displays the electronlike band and the surface states. The Dirac point lies about 375 meV below the Fermi level. (b) Resistance and magnetization measurements of the sample measured in the Shubnikov-de Hass part. The sharp resistive transition at $2.7 \mathrm{~K}$, along with the large superconducting volume fraction, clearly demonstrates a 3D bulk superconductivity. (c) Hall measurements at various temperatures. The linearity of the Hall coefficient with respect to the magnetic field is visible, while a clear temperature dependence is apparent. Inset: The carrier density inferred from the Hall measurements vs temperature.

This finding is strong evidence of an open FS, as two frequencies will show up only if the FS has two extrema with different cross-sectional areas. Therefore, a closed Fermi surface would have two frequencies only for a complicated shape, which is inconsistent with previous ARPES measurements and theoretical density functional theory (DFT) and $k \cdot p$ calculations $[18,25]$.

The two frequencies persist over a wide temperature range. However, as we increase $\theta$, only one frequency remains visible in the $\mathrm{SdH}$ data beyond $10^{\circ}$. The remaining frequency increases when rotating the field away from the $c$ axis, becoming unresolved for $\theta>57^{\circ}$. This frequency is well described by $1 / \cos (\theta)$, as expected for an open cylindrical-type FS (see Fig. 2). A possible explanation for the rapid disappearance of the second frequency is field-assisted tunneling between adjacent semiclassical orbits, similar to the magnetic breakdown phenomena [26,27]. To account for the decrease in the higher frequency, we have to assume a rather sharp corrugation on top of an open cylindrical-like Fermi surface. This FS results in two semiclassical orbits that are separated in the $k_{z}$ direction, but the shorter one spans a larger portion of the Brillouin zone. At an out-of-plane magnetic field the Lorentz force is confined in plane, but upon increasing the tilt angle, the Lorentz force acquires a $k_{z}$ component, allowing the semiclassical electron to scatter to the shorter orbit. Furthermore, the tilting of the magnetic field brings the two semiclassical orbits closer in $k$ space, making the tunneling between the orbits even more probable.

\section{B. ARPES measurements}

Due to the larger uncertainties for $\theta>50^{\circ}$, we found it necessary to support our analysis suggesting an open FS in $\mathrm{Sr}_{x} \mathrm{Bi}_{2} \mathrm{Se}_{3}$. We turn to ARPES measurements in which we vary the photon energy and thus scan the entire Brillouin zone along the $k_{z}$ direction $[19,28,29]$. The inner potential that determines the conversion of photon energy to momentum normal to the surface is derived in Appendix E.

Each panel in Fig. 3 displays the dispersion of the conduction band with respect to the momentum parallel to the surface for different photon energies. We find intensity corresponding to the bulk band for all measured $k_{z}$ values, including the $\Gamma$ and $Z$ points. Our data showing a finite density of states at both $Z$ points support our conclusion of an open FS. For comparison, we note that no intensity is seen by ARPES at $k_{z}$ corresponding to the $Z$ points for low carrier density samples of $\mathrm{Bi}_{2} \mathrm{Se}_{3}$ having a closed FS [19,30]. The bandwidth changes with $k_{z}$ and is maximal at the $\Gamma$ point, similar to the case of $\mathrm{Cu}_{x} \mathrm{Bi}_{2} \mathrm{Se}_{3}$ and consistent with our $\mathrm{SdH}$ data. Furthermore, the Fermi momentum is independent of $k_{z}$, suggesting a nearly perfect cylinder. The constant Fermi momentum in the $\mathrm{k}_{\mathrm{x}}-\mathrm{k}_{\mathrm{y}}$ plane for different $k_{z}$ suggests that the effective mass of the band depends on $k_{z}$ (see Appendix G).

\section{Carrier density estimation}

The temperature and intercalation dependence of the Hall coefficient casts doubt on whether it can be used for 

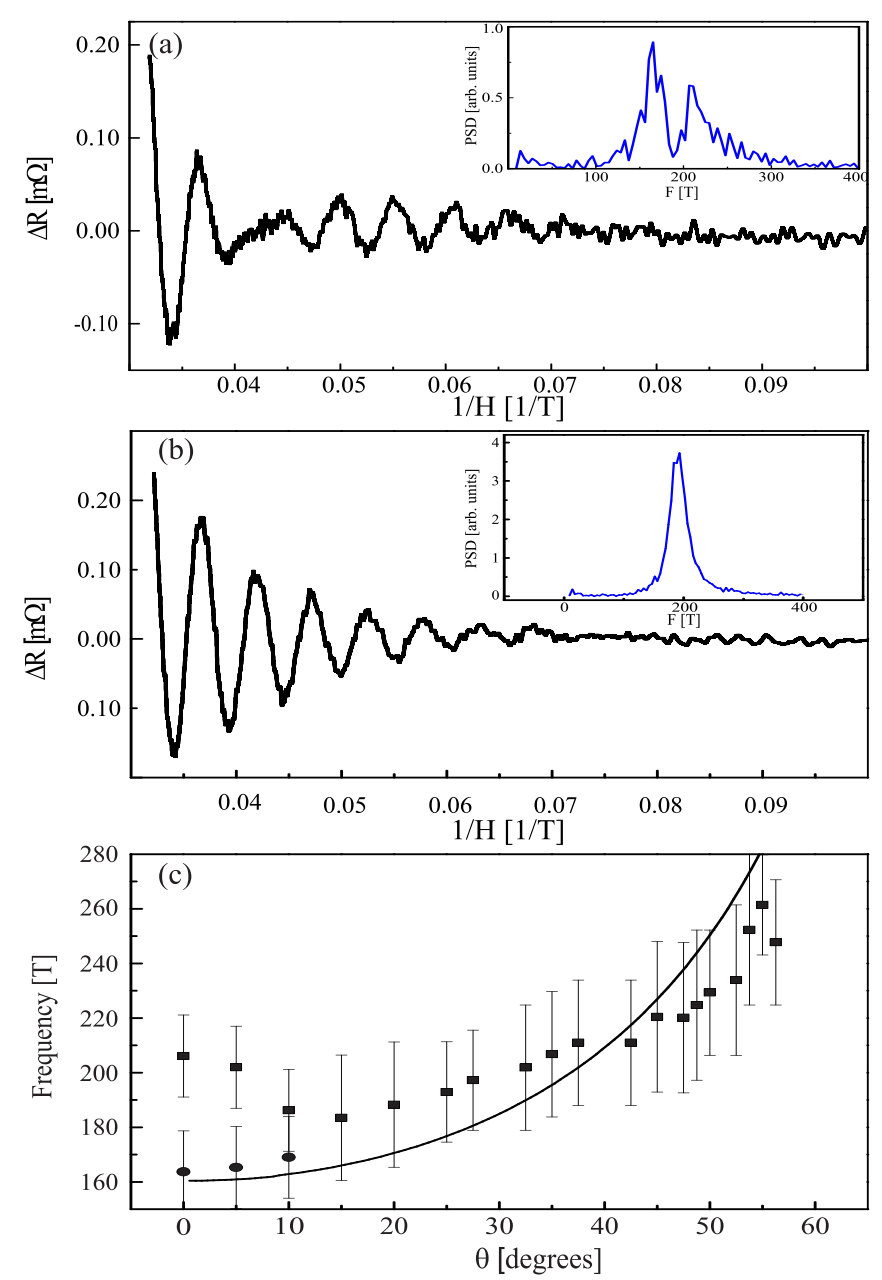

FIG. 2. Angular dependence of the SdH frequency at $0.4 \mathrm{~K}$. (a) Two frequencies are observed for $\theta \simeq 0^{\circ}$. The two frequencies point to an open Fermi surface, as explained in the text. (b) As we rotate the sample to larger out-of-plane angles, we observe only one frequency above a certain angle, as can be seen, for example, at $\theta \simeq 20^{\circ}$. (c) $\mathrm{SdH}$ frequency as a function of angle. The solid line represents the expected angular dependence of one $\mathrm{SdH}$ frequency for an open cylinder. The insets in (a) and (b) are the Fourier power spectrum of the quantum oscillations vs inverse magnetic field $(1 / H), \theta$ is the angle between the magnetic field direction and the $c$ axis.

accurate determination of the carrier density. The Hall number is almost independent of $\mathrm{Sr}$ intercalation, although this intercalation is the main mechanism for doping mobile electrons in the sample $[23,31]$. Together with the unexpected large temperature dependence of the Hall number we conclude that the Hall-inferred density is unreliable. Doped $\mathrm{Bi}_{2} \mathrm{Se}_{3}$ has a single parabolic band close to the Fermi energy, and if we assume that the $\mathrm{SdH}$ signal originates only from the conducting bulk, the interpretation of the data is straightforward and gives exact information about the volume of the Fermi surface and the carrier density. We therefore revisit our previous results and calculate the carrier density from the volume in momentum space as inferred from ARPES and SdH. The Fermi momentum from ARPES yields $n=6.5 \pm 1 \times 10^{19} \mathrm{~cm}^{-3}$ for a noncorrugated cylinder. The $\mathrm{SdH}$ numbers for this sample and for other samples are summarized in Table I.
TABLE I. Comparison between the Hall carrier density and the $\mathrm{SdH}$ carrier density.

\begin{tabular}{lccc}
\hline \hline Sample & $n_{S d H}\left(\mathrm{~cm}^{-3}\right)$ & $n_{\text {Hall }}\left(\mathrm{cm}^{-3}\right)$ & Ref. \\
\hline $\mathrm{Bi}_{2} \mathrm{Se}_{3}$ (not SC) & $1.9 \times 10^{18}$ & $3.7 \times 10^{17}$ & {$[19]$} \\
$\mathrm{Bi}_{2} \mathrm{Se}_{3}$ (not SC) & $1.8 \times 10^{19}$ & $2.3 \times 10^{19}$ & {$[19]$} \\
$\mathrm{Cu}_{x} \mathrm{Bi}_{2} \mathrm{Se}_{3}$ (SC) & $1.6 \times 10^{20}$ & $2.7 \times 10^{20}$ & {$[19]$} \\
$\mathrm{Sr}_{x} \mathrm{Bi}_{2} \mathrm{Se}_{3}$ (SC) & $9.3 \times 10^{19}$ & $2 \times 10^{19 \mathrm{a}}$ & This paper \\
\hline \hline
\end{tabular}

a The Hall density of $\mathrm{Sr}_{x} \mathrm{Bi}_{2} \mathrm{Se}_{3}$ is taken at $T=6 \mathrm{~K}$.

A similar Fermi surface topology is found in all intercalated $\mathrm{Bi}_{2} \mathrm{Se}_{3}$ samples exhibiting superconductivity [19,32]. This observation raises the question of why superconductivity appears concomitantly with an open Fermi surface.

\section{Theoretical model for Tc vs. Fermi surface topology}

Intuitively, one might expect that the opening of the FS will result in a quasi-2D band structure that has a larger density of states than the $3 \mathrm{D}$ case for same carrier density. In the framework of weak-coupling BCS theory, the increase in the density of states would enhance the critical temperature $T_{c}$. We use a simple model to quantify this intuitive idea, using weak-coupling-limit BCS theory and the band structure of $\mathrm{Bi}_{2} \mathrm{Se}_{3}$, based on DFT calculations for pure $\mathrm{Bi}_{2} \mathrm{Se}_{3}[2,33]$ (see Appendix $\mathrm{F}$ for details). To account for the effect of the intercalation the coupling amplitudes in the $\hat{z}$ direction are varied as a function of the chemical potential [25]. The FS of this band structure is a narrow, ellipsoid closed surface at low carrier densities and a cylindrical open surface at high carrier densities, as shown in Fig. 4 and in agreement with the experimental observation.

We can gauge the effect of the Lifshitz transition by plugging the density of states $g(\mu)$ into the standard weakcoupling BCS [34] expression for the critical temperature $T_{c}$ in the weak-coupling regime:

$$
k_{B} T_{c} \approx 1.14 \hbar \omega_{D} \exp \left(-\frac{1}{V g(\mu)}\right),
$$

where $\omega_{D}$ is the Debye frequency and $V$ is the electronphonon coupling constant. We use the known $\omega_{D}$ and adjust $V$ to match the critical temperature of the system at large enough carrier densities where $g(\mu)$ is approximately constant. Assuming that $V$ is independent of carrier density, we can plug it back into Eq. (1) and estimate $T_{c}$ for different carrier densities. As shown in Fig. 4, the critical temperature of the open FS $\left(n>5 \times 10^{19} \mathrm{~cm}^{-3}\right)$ is approximately constant but drops sharply at carrier densities below the Lifshitz transition once the Fermi surface closes. Two experimental points $T_{c}(n)$ are indicated on the plot, matching the critical temperatures and carrier densities for $\mathrm{Cu}_{x} \mathrm{Bi}_{2} \mathrm{Se}_{3}$ and $\mathrm{Sr}_{x} \mathrm{Bi}_{2} \mathrm{Se}_{3}$ deduced from $\mathrm{SdH}$ measurements.

\section{CONCLUSIONS}

The main finding is that superconducting samples always have large $\left(\geqslant 6.5 \times 10^{19} \mathrm{~cm}^{-3}\right)$ carrier densities, which results in an open FS, consistent with our simplified model 


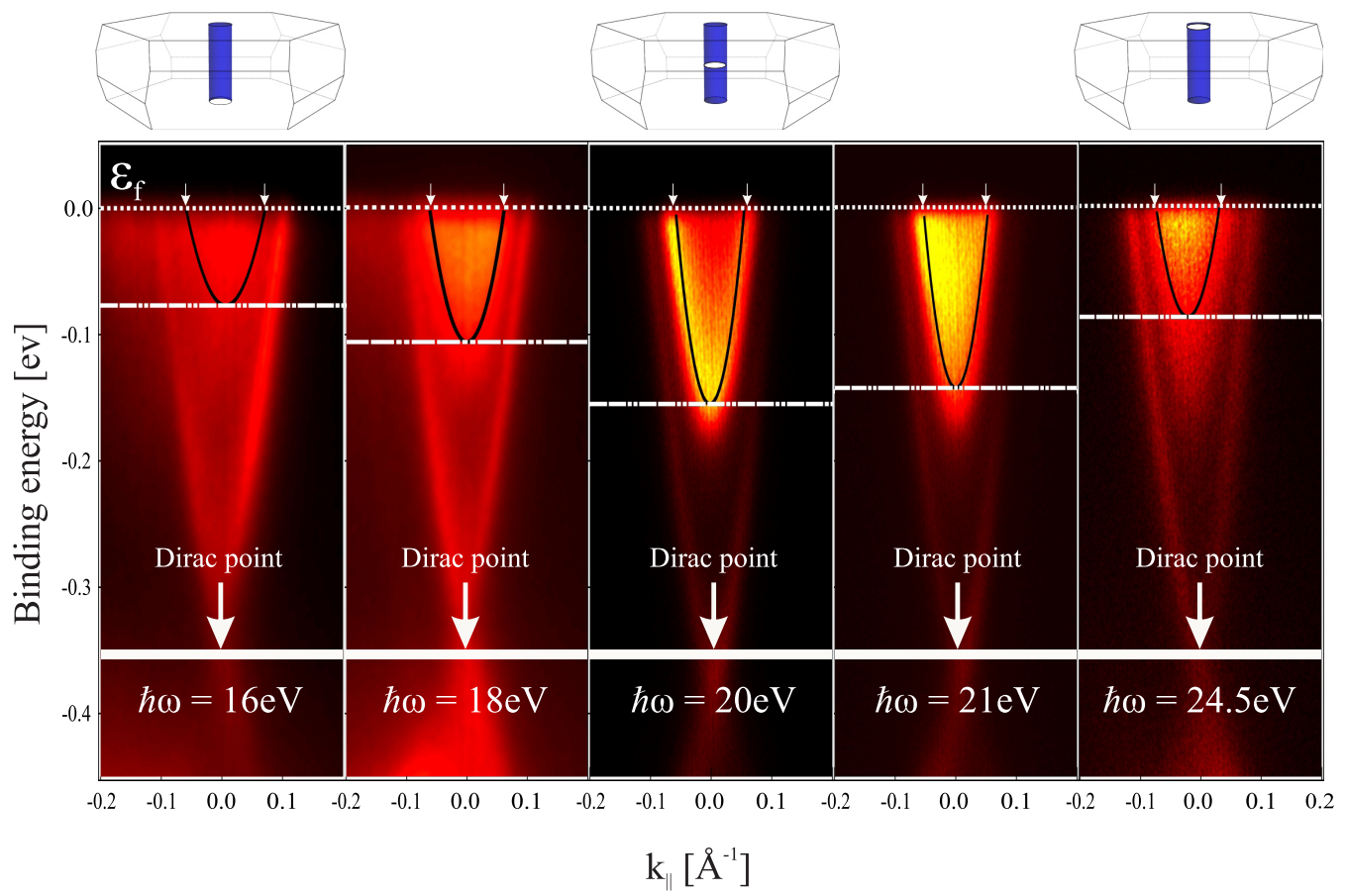

FIG. 3. Band structure of $\mathrm{Sr}_{x} \mathrm{Bi}_{2} \mathrm{Se}_{3}$ samples measured at various photon energies; each photon energy corresponds to a different $k_{z}$ value (see Appendix E). The two outermost panels are measurements at the two $Z$ points, while the middle panel is a measurement at $k_{z}=0$. The 16 and $18 \mathrm{eV}$ data were measured at the BaDElPh beam line at Elettra, and the 20, 21, and $24.5 \mathrm{eV}$ data were measured at the Surface/Interface Spectroscopy (SIS) beamline at the Swiss Light Source, PSI. The small arrows mark the Fermi momentum, and the large arrow marks the Dirac point. The Fermi level and the minimum of the band are marked by dashed and dash-dotted lines, respectively. In all panels the energy of the Dirac point is the same, as expected. The drawings on top show an illustration of the constant $k_{z}$ plane at which the spectra in the panel below it were measured.

and measurements done using different techniques. From the theoretical point of view [17], a 3D topological superconduc-

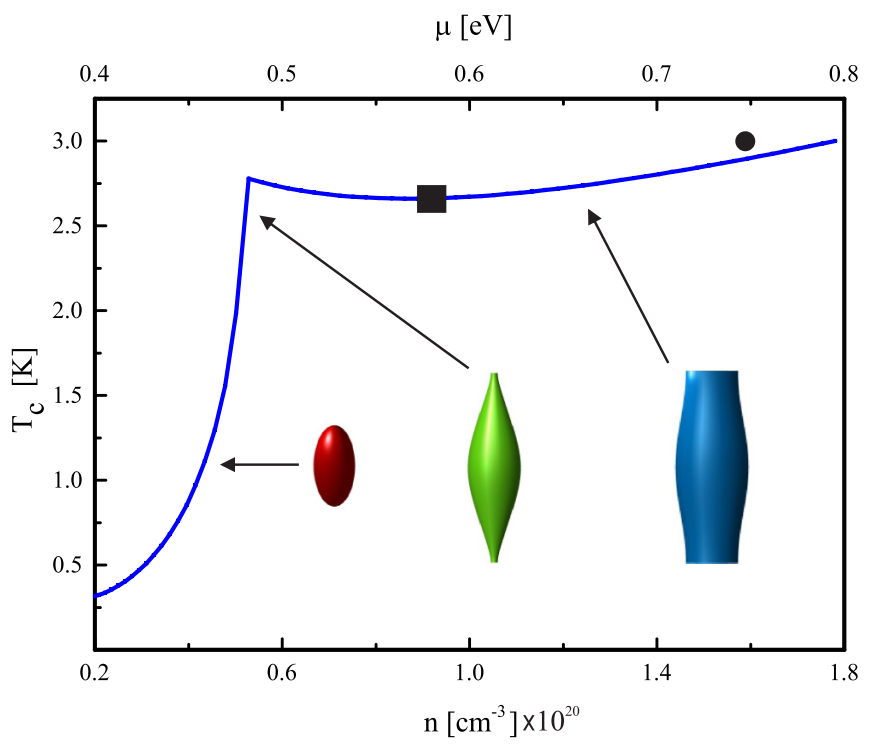

FIG. 4. Theoretical chemical potential/carrier density dependence of the superconducting critical temperature, obtained by combining DFT-based band structure with the BCS theory. Note the chemical potential calculated is about three times larger than the maximum bandwidth obtained by ARPES. Two experimental points are presented, a black dot for $\mathrm{Cu}_{x} \mathrm{Bi}_{2} \mathrm{Se}_{3}$ [19] and a black square for $\mathrm{Sr}_{x} \mathrm{Bi}_{2} \mathrm{Se}_{3}$, showing the consistency of the theoretical model. tor is not achievable with our suggested Fermi surface. This still leaves the door open for other scenarios of weak 2D topological superconductivity [35].

An open Fermi surface is consistent with two more observations. First, the most realistic physical mechanism of superconducting pairing in $\mathrm{Sr}_{x} \mathrm{Bi}_{2} \mathrm{Se}_{3}$ is the electronphonon interaction, which occurs at small phonon momenta [14,36]. An open Fermi surface allows this mechanism to be

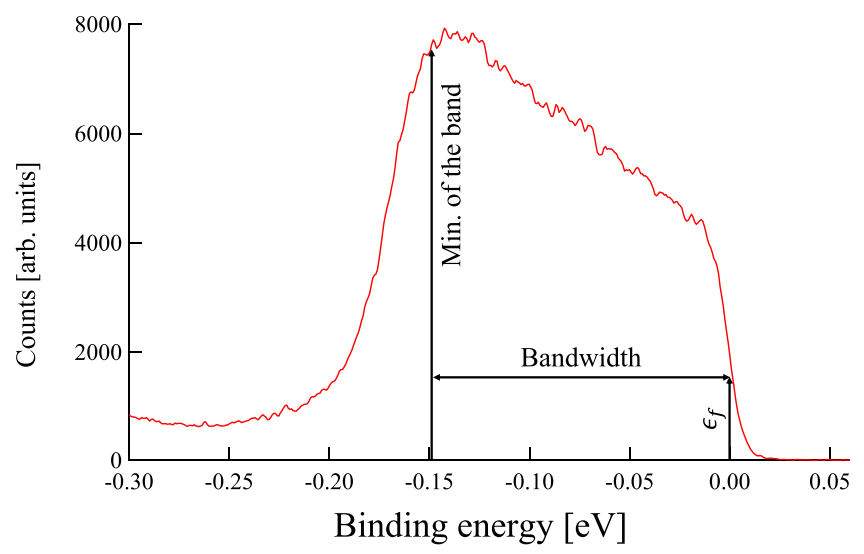

FIG. 5. EDC at $k_{\|}=0$ ( $\Gamma$ point) used to identify the Fermi energy. The exponential-like increase of the counts is observed at the energy range -0.25 to $-0.15 \mathrm{eV}$. We define the bandwidth as the energy difference between the end of the exponential-like increase and the Fermi level. 


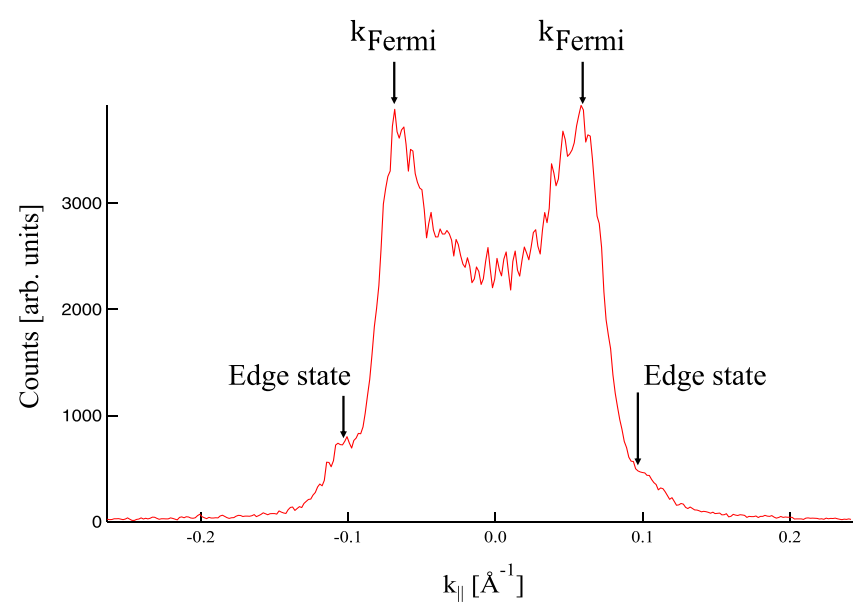

FIG. 6. Momentum dispersive curve taken at the Fermi level. The Fermi momentum points are marked by arrows. Also marked are the edge states, with lower counts than the bulk band.

energetically favorable [14], as confirmed recently by inelastic neutron scattering [37]. Second, as shown in Ref. [38], for an odd-parity $E_{u}$ pairing, the nodeless superconducting gap observed in tunneling measurements in $\mathrm{Cu}_{x} \mathrm{Bi}_{2} \mathrm{Se}_{3}[2,4,5]$ and $\mathrm{Sr}_{x} \mathrm{Bi}_{2} \mathrm{Se}[39]$ is consistent only with an open Fermi surface.

In addition, the suggested mean-field picture with very weak dependence of $T_{c}$ on the density is in agreement with the observation of a Hall density independent $T_{c}$ in Cu-codoped $\mathrm{Sr}_{x} \mathrm{Bi}_{2} \mathrm{Se}_{3}$ crystals [22].

Finally, we note that according to our simplified model, a small region in the carrier density $\left(4-5 \times 10^{19} \mathrm{~cm}^{3}\right)$ versus temperature phase diagram may allow for $3 \mathrm{D}$ topological superconductivity. This would require precise tuning of carrier density, possibly using gate voltage or proper codoping.

\section{ACKNOWLEDGMENTS}

ARPES experiments were conducted at the Surface/Interface Spectroscopy (SIS) beamline of the Swiss Light Source at the Paul Scherrer Institute in Villigen, Switzerland, and at the BaDElPh beamline of Elettra. The high magnetic field measurements were performed at the National High Magnetic Field Laboratory, which is funded by the National Science Foundation through Grant No. DMR-1157490 and the U.S. Department of Energy and the state of Florida. The work on the Russian site (crystal growth and magnetotransport measurements) was supported by the Russian Science Foundation (Grant No. 17-12-01544). Magnetotransport measurements were performed using the equipment of the Lebedev Physical Institute (LPI) shared facility center. Work at Tel Aviv University and at Technion is supported by the Israeli Science Foundation under Grants No. 382/17 and No. 320/17 respectively. Theoretical work is supported by the Israeli Science Foundation (Grant No. 227/15) and the U.S.-Israel Binational Science Foundation (Grant No. 2016224). We acknowledge useful discussions with A. Auerbach.

A.A. and I.S. contributed equally to this work.

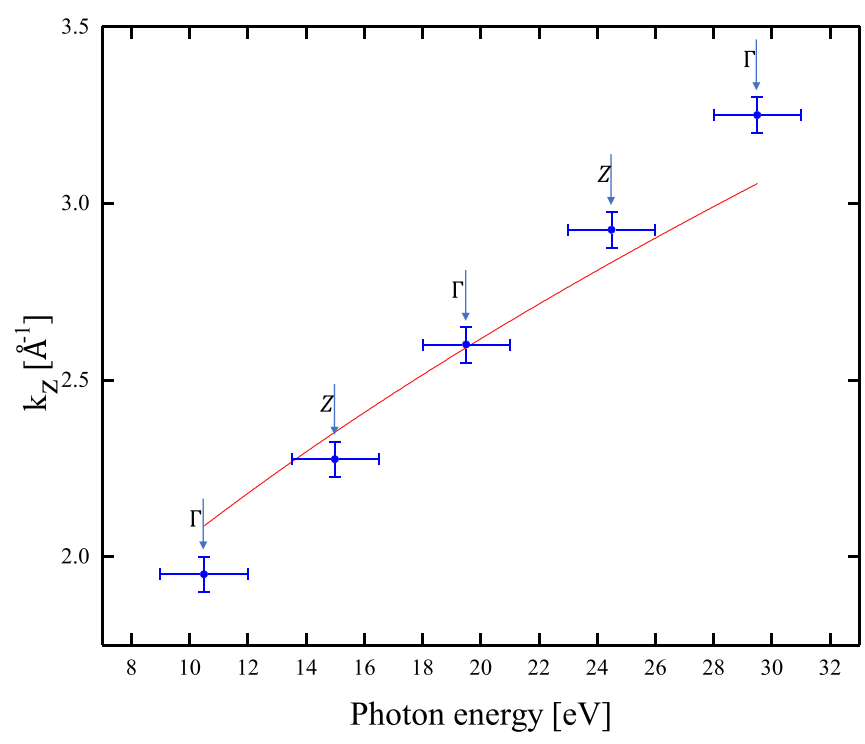

FIG. 7. A fit of the Brillouin zone high-symmetry point locations, $\Gamma$ and $Z$, to the photon energy. Using the free electron final state approximation, we extract the inner potential, $V_{0} \approx 10 \mathrm{~V}$, and map all of the scans to their respective $k_{z}$ values. The red line is the fit using the extracted inner potential; the blue points with the error bars represent the locations of the $\Gamma$ and $Z$ points and the uncertainty in photon energy and $k_{z}$ value.

\section{APPENDIX A: SAMPLE PREPARATION}

Our samples are single crystals grown using the modified Bridgman method. Elemental $\mathrm{Bi}, \mathrm{Se}$, and $\mathrm{Sr}$ were loaded in quartz ampules in a glove box with an inert environment and sealed in evacuated tubes. More details on the growing process are described elsewhere [7,23]. Samples with lateral dimensions of $0.5-5 \mathrm{~mm}^{2}$ were cleaved from the boule. All samples have a full volume fraction, as measured by magnetization curves [see Fig. 1(b) in the main text], for the data taken down to $1.7 \mathrm{~K}$, where the volume fraction is $\approx 70 \%$, as expected from previous measurements. Extrapolating the data down to $0 \mathrm{~K}$ gives a volume fraction of $100 \%$. Resistivity measurements give $T_{C}=2.77 \mathrm{~K}$, as defined by the onset of the transition, with a width of $\approx 0.2 \mathrm{~K}$ [7]. We denote the nominal Sr content $x_{\text {nominal }}$ because its real content $(\sim 0.06)$ is systematically smaller than the nominal value (in our case $0.15)$ [31].

The crystals were previously shown to consist of blocks (with dimensions of several hundred micrometers) with slight mutual misorientation [7]. Since the properties of the neighboring blocks may slightly vary, for magneto-oscillation studies done at the Russian site we cleaved blocks as small as possible and glued the contacts with silver paint. The blocks were cleaved along the basal plane and had mirrorlike top

TABLE II. Model parameters (in eV), taken from Refs. [2,25].

\begin{tabular}{lccccccc}
\hline \hline$\mu$ & $\bar{D}_{1}$ & $\bar{D}_{2}$ & $M_{0}$ & $\bar{B}_{1}$ & $\bar{B}_{2}$ & $\bar{A}_{1}$ & $\bar{A}_{2}$ \\
\hline 0.4 & 0.024 & \multirow{2}{*}{1.14} & 0.28 & 0.216 & 3.30 & 0.32 & 0.99 \\
0.65 & 0.012 & & & 0.108 & & 0.16 & \\
\hline
\end{tabular}



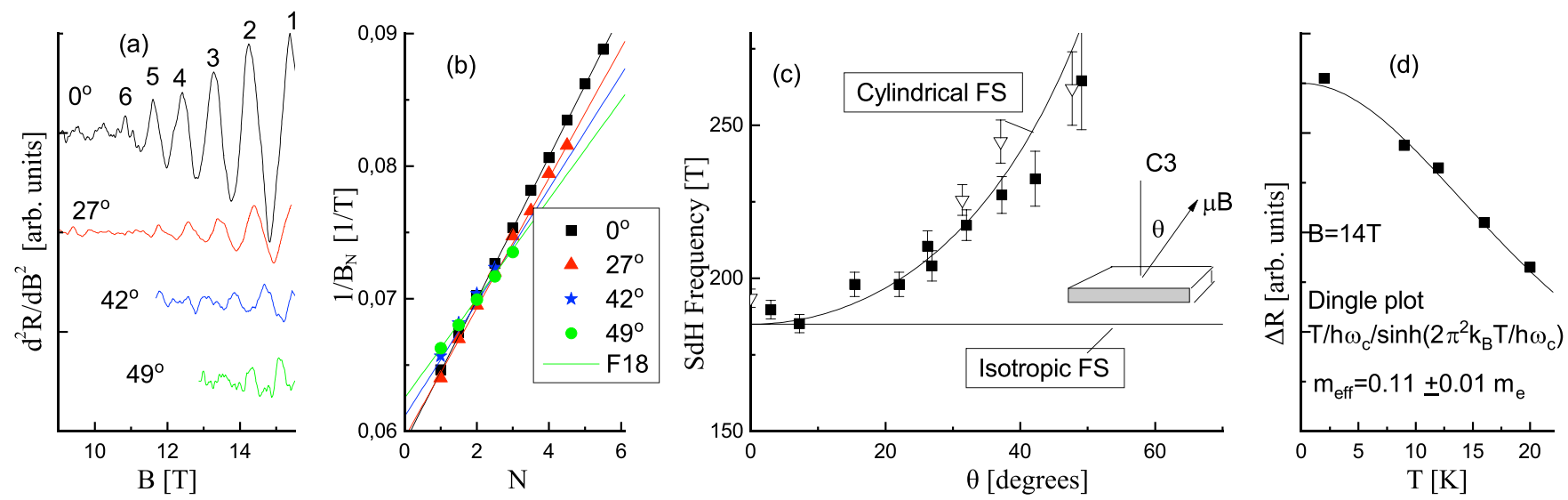

FIG. 8. (a) Shubnikov-de Haas oscillations for $\mathrm{Sr}_{0.1} \mathrm{Bi}_{2} \mathrm{Se}_{3}$ taken for different $\theta$. The maxima of oscillations $(\theta=0$ case $)$ are enumerated for frequency calculation. (b) Fan diagrams for the corresponding data sets from (a). (c) Frequencies of the SdH oscillations versus tilt angle. The geometry of the experiment is shown. Lines show the expectations in two limits: cylindrical and spherical Fermi surfaces, respectively. (d) Temperature dependence of the Shubnikov-de Haas oscillation amplitude at $14 \mathrm{~T}$ (magnetic field is perpendicular to the basal plane). The line is a fit to Lifshits-Kosevich formula (see text).

and bottom surfaces. Magneto-oscillation studies done at the National High Magnetic Field Laboratory in Florida were done using cured silver epoxy contacts with Pt wires.

\section{APPENDIX B: ARPES MEASUREMENTS}

ARPES measurements were done at the SIS beamline at the Swiss Light Source (SLS) at the Paul Scherrer Institute (Switzerland) and at BaDElPh beamline at Elettra (Italy). Samples at the SLS were cleaved and measured at $T=12 \mathrm{~K}$ and in vacuum better than $5 \times 10^{-11}$ Torr, with photon energy increments of $0.5 \mathrm{eV}$, ranging from 20 to $35 \mathrm{eV}$. Samples at Elettra were cleaved and measured at $T=28.5 \mathrm{~K}$ and in vacuum better than $5 \times 10^{-11}$ Torr, with photon energy increments of $0.5 \mathrm{eV}$, ranging from 15 to $24 \mathrm{eV}$. The energy resolution is $\sim 10 \mathrm{meV}$.

\section{APPENDIX C: TRANSPORT MEASUREMENTS}

$R(T)$ measurements were done in a home-built $\mathrm{He}_{4}$ cryostat, with electrical current of $0.5 \mathrm{~mA}$. A Keithley 6221 supplied the current, and the voltage drop was recorded with a Keithley 2182A nanovoltmeter in the delta mode configuration. Hall measurements were measured with a Quantum Design physical property measurement system using the same delta mode configuration while applying a larger current of $1 \mathrm{~mA}$. The data are presented after averaging two perpendicular $R_{x y}$ configurations and symmetrization with respect to positive and negative applied magnetic fields.

The high-field measurements were taken with a wet $\mathrm{He}_{3}$ cryostat at the National High Magnetic Field Laboratory in Tallahassee, Florida. The maximum field of $31 \mathrm{~T}$ was ramped up at a typical scan rate of $1.5 \mathrm{~T} / \mathrm{min}$. Resistance was measured using a Lakeshore 372 resistance bridge with a current of $316 \mu \mathrm{A}$. The out-of-plane angle was determined by the brushless stepper motor encoder while keeping the sweep direction constant in order to avoid any mechanical backlash, recoil arising between parts of a mechanism. The stepper motor and rotator platform were tested the day prior to the measurements by measuring the critical field of a 2D superconducting $4 H_{b}-\mathrm{TaS}_{2}$ sample at different angles.

\section{APPENDIX D: BAND DISPERSION DETERMINATION}

We define the bottom of the band as a point were an exponential-like increase of intensity intersects with a linear trend of the intensity. We use an average of six energy dispersive curves (EDCs) located around $k_{\|}=0$ (Fig. 5). We define the Fermi momentum as the start of a prominent decrease in intensity, when diverging from $k_{\|}=0$ towards higher absolute values of $k_{\|}=0$ (Fig. 6). This is done using a momentum dispersive curve located slightly below the Fermi energy $\left(E \sim E_{f}\right)$.

\section{APPENDIX E: BAND DISPERSION ALONG $\boldsymbol{k}_{z}$}

To resolve $k_{z}$ values we use the free electron final state approximation [19,29,40]: $k_{z}=\sqrt{2 m^{*} / \hbar^{2}\left(E_{\mathrm{kin}}+V_{0}\right)}$. The mapping of band dispersion in the $k_{z}$ direction is then carried out by changing the photon energy used in the photoemission process, thus changing the value of $E_{\text {kin }}$ and obtaining a different value for $k_{z}$. The constant $V_{0}$ is specific to the material and is called the inner potential; formally, it is given by $V_{0}=\mu+\phi$, where $\mu$ is the chemical potential measured from the bottom of the band and $\phi$ is the work function. By fitting the photon energies of the high-symmetry points of the Brillouin zone ( $\Gamma$ and $Z$ ) and using the known values of $k_{z}$ for those points, we can extract the inner potential. In Figure 7 we plot the position of the high-symmetry points as a function of photon energy and obtain a value of approximately $V_{0} \sim 10 \mathrm{eV}$.

\section{APPENDIX F: THEORETICAL CALCULATIONS OF THE BCS MODEL}

The calculation of the Fermi surface and the electronic density of states (DOS) is based on an effective tight-binding low-energy Hamiltonian obtained for clean $\mathrm{Bi}_{2} \mathrm{Se}_{3}$ from DFT calculations [2,33], introducing a chemical potential 
dependence in several of the parameters in order to account for intercalation effects [25]. The Hamiltonian in the following equation is taken according to the Supplemental Material of Ref. [2]:

$$
\begin{aligned}
& H(\boldsymbol{k})=\left(\begin{array}{cccc}
\varepsilon(\boldsymbol{k})+M(\boldsymbol{k}) & & A_{1}(\boldsymbol{k}) & A_{2}(\boldsymbol{k}) \\
& \varepsilon(\boldsymbol{k})+M(\boldsymbol{k}) & A_{2}^{*}(\boldsymbol{k}) & -A_{1}(\boldsymbol{k}) \\
A_{1}(\boldsymbol{k}) & A_{2}(\boldsymbol{k}) & \varepsilon(\boldsymbol{k})-M(\boldsymbol{k}) & \\
A_{2}^{*}(\boldsymbol{k}) & -A_{1}(\boldsymbol{k}) & & \varepsilon(\boldsymbol{k})-M(\boldsymbol{k})
\end{array}\right), \\
& \varepsilon(\boldsymbol{k})=-\mu+\bar{D}_{1}\left[2-2 \cos \left(k_{z} c\right)\right] \\
& +\frac{4}{3} \bar{D}_{2}\left[3-2 \cos \left(\frac{\sqrt{3}}{2} k_{x} a\right) \cos \left(\frac{1}{2} k_{y} a\right)-\cos \left(k_{y} a\right)\right], \\
& M(\boldsymbol{k})=M_{0}-\bar{B}_{1}\left[2-2 \cos \left(k_{z} c\right)\right] \\
& -\frac{4}{3} \bar{B}_{2}\left[3-2 \cos \left(\frac{\sqrt{3}}{2} k_{x} a\right) \cos \left(\frac{1}{2} k_{y} a\right)-\cos \left(k_{y} a\right)\right], \\
& A_{1}(\boldsymbol{k})=\bar{A}_{1} \sin \left(k_{z} c\right) \text {, } \\
& A_{2}(\boldsymbol{k})=\frac{2}{3} \bar{A}_{2}\left\{\sqrt{3} \sin \left(\frac{\sqrt{3}}{2} k_{x} a\right) \cos \left(\frac{1}{2} k_{y} a\right)\right. \\
& \left.+i\left[\cos \left(\frac{\sqrt{3}}{2} k_{x} a\right) \sin \left(\frac{1}{2} k_{y} a\right)+\sin \left(k_{y} a\right)\right]\right\} .
\end{aligned}
$$

We take $a=4.14$ Åand note that here $c=9.58 \AA$ is the height of the primitive unit cell and not of the hexagonal lattice, as often appears in the literature. For a chemical potential $\mu=0.4 \mathrm{eV}$ the coefficients are taken as in [2]. Intercalation is expected to mostly affect the parameters characterizing the $k_{z}$ dependence, namely, $\bar{D}_{1}, \bar{B}_{1}, \bar{A}_{1}$. Thus, these components are chosen for $\mu=0.65 \mathrm{eV}$ as in Ref. [25] and interpolated linearly in between. For the convenience of the reader we list the values of all the parameters in Table II. Note that the qualitative behavior of the results is not sensitive to this interpolation.

Diagonalizing the Hamiltonian results in two spindegenerate bands, with the chemical potential in the gap between them:

$$
\xi_{ \pm}(\boldsymbol{k})=\varepsilon(\boldsymbol{k}) \pm \sqrt{M^{2}(\boldsymbol{k})+A_{1}^{2}(\boldsymbol{k})+\left|A_{2}(\boldsymbol{k})\right|^{2}} .
$$

Intercalation raises the carrier density and shifts the chemical potential into the (twofold-degenerate) upper band $\xi_{+}(\boldsymbol{k})$. The carrier density at the Fermi energy $\mu$ is then given by

$$
n(\mu)=2 \int_{B Z} \frac{d^{3} k}{(2 \pi)^{3}} \Theta\left(-\xi_{+}(\boldsymbol{k})\right) .
$$

Holding all parameters (including $\bar{D}_{1}, \bar{B}_{1}, \bar{A}_{1}$ ) constant for each $\mu$, we can numerically differentiate $n(\mu)$ and obtain the DOS $g(\mu)$, which we then plug into the BCS formula.

\section{APPENDIX G: ADDITIONAL DATA}

Additional SdH data were measured on a different sample from the same growth as described in the main text and also for the sample with the nominal composition $\mathrm{Sr}_{0.1} \mathrm{Bi}_{2} \mathrm{Se}_{3}$. The results for different samples were similar. $\mathrm{SdH}$ oscillations in magnetic fields up to $16 \mathrm{~T}$ are observed up to $\theta \approx 55^{\circ}$ [see Fig. 8(a)]. Due to lower available magnetic field, a fast

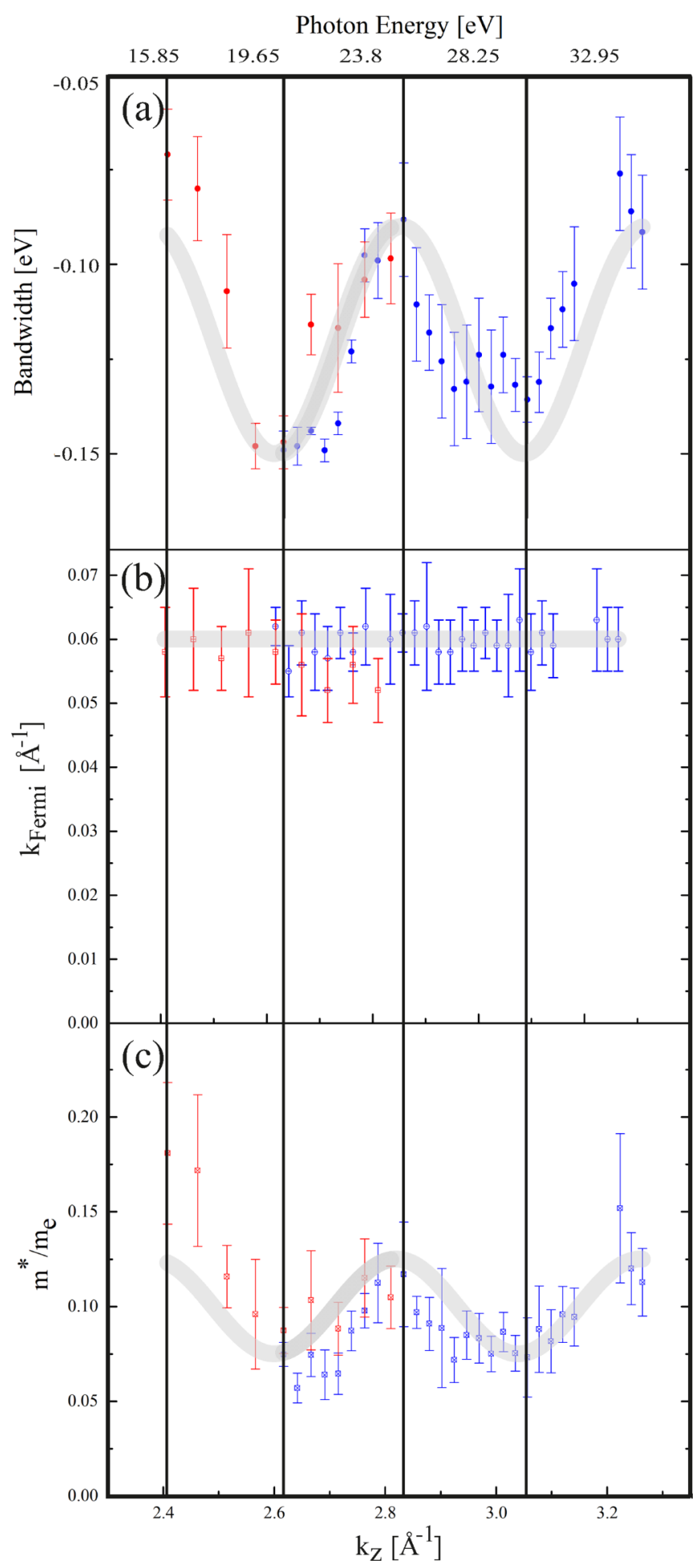

FIG. 9. (a) Bandwidth, (b) Fermi momentum, and (c) relative effective mass extracted for different $k_{z}$ values on the $Z-\Gamma-Z$ line in the Brillouin zone, as described in the text. The $k_{z}$ values were extracted using the inner potential and the free electron state approximation. The blue dots represent data measured at PSI on the same sample. Red dots represent data measured at Elettra on a different sample. An open Fermi surface is seen from the fact that the Fermi momentum is unchanged up to the $Z$ point and that the bandwidth is nonzero at every $k_{z}$ value. Thick gray lines are guides to the eye. 
Fourier transform procedure was replaced with a minimamaxima counting procedure to evaluate the frequency at each angle [Fig. 8(b)]; the linear relation observed between the order of the maxima to $1 / \mathrm{H}$ clearly shows the strength of this procedure, although it obscures the two frequencies observed at low angles. This data analysis reinforced our conclusion of an open FS in these samples based on the fit of the results to the known $1 / \cos (\theta)$ relation for an open-cylindrical FS [Fig. 8(c)]. The dingle plot presented in Fig. 8(d) gives an average effective mass with excellent agreement with the results acquired from the ARPES data (Fig. 9).

A summary of all the ARPES data from both the sample from the Paul Scherrer Institute (PSI; blue) and from Elettra (red) is presented in Fig. 9. The data span about two Brillouin zones and clearly show that an open FS is measured, as a finite bandwidth is visible in every scan. Figure 9(a) shows the bottom of the band as a function of $k_{z}$. A clear change in the bottom of the band, with values ranging from $\sim 150 \mathrm{meV}$ at the $\Gamma$ point to $\sim 80 \mathrm{meV}$ at the edge of the Brillouin zone ( $Z$ point), is shown. Each photon energy is a cut at different $k_{z}$; thus, a closed FS would have zero intensity at the edge of the Brillouin zone.

Figure 9(b) shows the Fermi momentum as a function of $k_{z}$. A constant Fermi momentum of $\sim k_{\|}=0.062 \pm 0.005 \AA^{-1}$ is observed at each $k_{z}$ value, indicating an open cylinderlike FS. Due to the resolution, we were unable to determine whether the FS is a perfect cylinder or a corrugated one, as implied by the SdH results. A cylindrical FS with a constant Fermi momentum suggests that the effective mass depends on $k_{z}$, as seen in Fig. 9(c). An open cylindrical FS with changing effective mass was seen previously in the closely related material $\mathrm{Cu}_{x} \mathrm{Bi}_{2} \mathrm{Se}_{3}[19]$.
[1] Y. S. Hor, A. J. Williams, J. G. Checkelsky, P. Roushan, J. Seo, Q. Xu, H. W. Zandbergen, A. Yazdani, N. P. Ong, and R. J. Cava, Phys. Rev. Lett. 104, 057001 (2010).

[2] S. Sasaki, M. Kriener, K. Segawa, K. Yada, Y. Tanaka, M. Sato, and Y. Ando, Phys. Rev. Lett. 107, 217001 (2011).

[3] T. Kirzhner, E. Lahoud, K. B. Chaska, Z. Salman, and A. Kanigel, Phys. Rev. B 86, 064517 (2012).

[4] N. Levy, T. Zhang, J. Ha, F. Sharifi, A. A. Talin, Y. Kuk, and J. A. Stroscio, Phys. Rev. Lett. 110, 117001 (2013).

[5] R. Tao, Y.-J. Yan, X. Liu, Z.-W. Wang, Y. Ando, Q.-H. Wang, T. Zhang, and D.-L. Feng, Phys. Rev. X 8, 041024 (2018).

[6] K. Matano, M. Kriener, K. Segawa, Y. Ando, and G.-Q. Zheng, Nat. Phys. 12, 852 (2016).

[7] A. Y. Kuntsevich, M. Bryzgalov, V. Prudkoglyad, V. Martovitskii, Y. G. Selivanov, and E. Chizhevskii, New J. Phys. 20, 103022 (2018).

[8] A. Y. Kuntsevich, M. A. Bryzgalov, R. S. Akzyanov, V. P. Martovitskii, A. L. Rakhmanov, and Y. G. Selivanov, Phys. Rev. B 100, 224509 (2019).

[9] S. Yonezawa, K. Tajiri, S. Nakata, Y. Nagai, Z. Wang, K. Segawa, Y. Ando, and Y. Maeno, Nat. Phys. 13, 123 (2017).

[10] K. Willa, R. Willa, K. W. Song, G. D. Gu, J. A. Schneeloch, R. Zhong, A. E. Koshelev, W.-K. Kwok, and U. Welp, Phys. Rev. B 98, 184509 (2018).

[11] Y. Sun, S. Kittaka, T. Sakakibara, K. Machida, J. Wang, J. Wen, X. Xing, Z. Shi, and T. Tamegai, Phys. Rev. Lett. 123, 027002 (2019)

[12] T. Asaba, B. J. Lawson, C. Tinsman, L. Chen, P. Corbae, G. Li, Y. Qiu, Y. S. Hor, L. Fu, and L. Li, Phys. Rev. X 7, 011009 (2017).

[13] L. Fu, Phys. Rev. B 90, 100509(R) (2014).

[14] X. Wan and S. Y. Savrasov, Nat. Commun. 5, 4144 (2014).

[15] J. W. F. Venderbos, V. Kozii, and L. Fu, Phys. Rev. B 94, 180504(R) (2016).

[16] H. S. Røising, F. Flicker, T. Scaffidi, and S. H. Simon, Phys. Rev. B 98, 224515 (2018).

[17] L. Fu and E. Berg, Phys. Rev. Lett. 105, 097001 (2010).

[18] W. Zhang, R. Yu, H.-J. Zhang, X. Dai, and Z. Fang, New J. Phys. 12, 065013 (2010).
[19] E. Lahoud, E. Maniv, M. S. Petrushevsky, M. Naamneh, A. Ribak, S. Wiedmann, L. Petaccia, Z. Salman, K. B. Chashka, Y. Dagan, and A. Kanigel, Phys. Rev. B 88, 195107 (2013).

[20] B. J. Lawson, G. Li, F. Yu, T. Asaba, C. Tinsman, T. Gao, W Wang, Y. S. Hor, and L. Li, Phys. Rev. B 90, 195141 (2014).

[21] Shruti, V. K. Maurya, P. Neha, P. Srivastava, and S. Patnaik, Phys. Rev. B 92, 020506(R) (2015).

[22] A. Y. Kuntsevich, V. P. Martovitskii, G. V. Rybalchenko, Y. G. Selivanov, M. I. Bannikov, O. A. Sobolevskiy, and E. G. Chigevskii, Materials 12, 3899 (2019).

[23] S. Volosheniuk, Y. G. Selivanov, M. Bryzgalov, V. Martovitskii, and A. Y. Kuntsevich, J. Appl. Phys. 125, 095103 (2019).

[24] L. Onsager, London, Edinburgh, Dublin Philos. Mag. J. Sci. 43, 1006 (1952).

[25] T. Hashimoto, K. Yada, A. Yamakage, M. Sato, and Y. Tanaka, Supercond. Sci. Technol. 27, 104002 (2014).

[26] A. B. Pippard, Proc. R. Soc. London, Ser. A 270, 1 (1962).

[27] N. Harrison, J. Caulfield, J. Singleton, P. Reinders, F. Herlach, W. Hayes, M. Kurmoo, and P. Day, J. Phys.: Condens. Matter 8, 5415 (1996).

[28] S.-Y. Xu, Y. Xia, L. A. Wray, S. Jia, F. Meier, J. H. Dil, J. Osterwalder, B. Slomski, A. Bansil, H. Lin, R. J. Cava, and M. Z. Hasan, Science 332, 560 (2011).

[29] S. Hufner, Photoelectron Spectroscopy: Principles and Applications (Springer-Verlag, Berlin, Heidelberg, 2013).

[30] Y. Tanaka, K. Nakayama, S. Souma, T. Sato, N. Xu, P. Zhang, P. Richard, H. Ding, Y. Suzuki, P. Das, K. Kadowaki, and T. Takahashi, Phys. Rev. B 85, 125111 (2012).

[31] Z. Liu, X. Yao, J. Shao, M. Zuo, L. Pi, S. Tan, C. Zhang, and Y. Zhang, J. Am. Chem. Soc. 137, 10512 (2015).

[32] B. J. Lawson, P. Corbae, G. Li, F. Yu, T. Asaba, C. Tinsman, Y. Qiu, J. E. Medvedeva, Y. S. Hor, and L. Li, Phys. Rev. B 94, 041114(R) (2016).

[33] H. Zhang, C.-X. Liu, X.-L. Qi, X. Dai, Z. Fang, and S.-C. Zhang, Nat. Phys. 5, 438 (2009).

[34] J. Bardeen, L. N. Cooper, and J. R. Schrieffer, Phys. Rev. 108, 1175 (1957).

[35] L. Fu and C. L. Kane, Phys. Rev. Lett. 100, 096407 (2008).

[36] P. M. R. Brydon, S. Das Sarma, H.-Y. Hui, and J. D. Sau, Phys. Rev. B 90, 184512 (2014). 
[37] J. Wang, K. Ran, S. Li, Z. Ma, S. Bao, Z. Cai, Y. Zhang, K. Nakajima, S. Ohira-Kawamura, P. Čermák, A. Schneidewind, S. Y. Savrasov, X. Wan, and J. Wen, Nat. Commun. 10, 2802 (2019).

[38] L. Hao and C. S. Ting, Phys. Rev. B 99, 214507 (2019).
[39] G. Du, J. Shao, X. Yang, Z. Du, D. Fang, J. Wang, K. Ran, J. Wen, C. Zhang, H. Yang, Y. Zhang, and H.-H. Wen, Nat. Commun. 8, 14466 (2017).

[40] A. Damascelli, Z. Hussain, and Z.-X. Shen, Rev. Mod. Phys. 75, 473 (2003). 